\title{
Low bit rate overhead based reference modification for error resilient video coding
}

\author{
Jun Wanga) and Satoshi Goto \\ Graduate School of Information Production, and systems, Waseda University, \\ Kitakyushu, 808-0135, Japan \\ a)wangjun@toki.waseda.jp
}

\begin{abstract}
During video transmission, errors may happen normally. Addressing error resiliency, the reference modification (RM) approach at video compression encoder is used to modify the original reference to a modified one, which is less sensitive to errors. Apparently it is a bit rate overhead compared to original reference based conventional encoder. Therefore to reduce such bit rate overhead while still keep the error resilience performance is a big issue. A low bit rate overhead based RM approach is proposed in this paper, where the decoder information is exploited to perform encoding. In the proposal, the modified reference has more correlation to original reference, thus can reduce the bit rate overhead. In addition, the proposal can achieve better image recovery compared to existing works, and still be compatible to standard decoder. Overall, the proposal provides the best trade-off among the error resiliency, coding efficiency, and standard compatibility.
\end{abstract}

Keywords: video coding, bit rate, error resilient

Classification: Electron devices, circuits, and systems

\section{References}

[1] T. Wiegand, et al., "Overview of the H.264/AVC video coding standard," IEEE Trans. Circuits Syst. Video Technol., pp. 560-576, 2003.

[2] Y. K. Wang, et al., "The error concealment feature in the H.26L test model," Proc. ICIP, vol. 2, pp. 729-732, 2002.

[3] [Online] http://iphome.hhi.de/suehring/tml

[4] Z. Li, et at., "Channel aware rate distortion optimized leaky motion prediction," Proc. ICIP, Oct. 2004.

[5] H. Yang, et al., "Source channel prediction in error resilient video coding," Proc. ICME, 2003.

[6] H. Yang, et al., "General aware rate distortion for error resilient video coding," Proc. ICASSP, 2006.

[7] A. Naghdinezhad, et al., "Reference frame modification methods in scalable video coding," Proc. MMSP, 2010.

[8] D. J. Conner, "Techniques for reducing the visibility of transmission errors in digitally encoded video signals," IEEE Trans. Commun., vol. 21. no. 6, pp. 695-706, 1973.

[9] H. Yang, et al., "Optimizing motion compensated prediction for error resilient video coding," IEEE Trans. Image Process., vol. 19. no. 1, 2010. 


\section{Introduction}

Reference frame is used as a motion predictor in a temporal estimation based video compression CODEC system including the state-of-the-art standard H.264/AVC [1]. Traditionally at the encoder side, the previous reconstructed frame is used as the predictor, while at the decoder side, the same one is supposed to be the predictor. In error free transmission, these 2 reference frames are the same. However, it is inevitable that error may happen in transmission, which produces the mismatch between the reference frames at the encoder and the decoder. Therefore the vision quality may degrade suffering error prone transmission.

There are lots of methods to stop or alleviate the vision quality degrading. For the decoder side, Error Concealment (EC) is proposed to recovery the damaged frame by employing the correlation to the survived information. One efficient and widely used EC approach is Boundary Match Algorithm (BMA) [2], which was suggested by H.264 in its reference software JM [3]. Another approach is to modify the reference block to a less vulnerable to error one. This approach is called Reference Modification (RM).

For RM, the modified reconstructed reference frame is usually formed as a weighted sum of original reference and "other values". There are lots of methods to produce the "other values". In leaky prediction (LP) of [4], it is formed by an appropriate constant. In source channel prediction (SCP) of [5], it is formulated by the previous collocated reconstructed block. The weighted sum of current reconstructed block and previous reconstructed one form a FIR filter to produce the modified reference. SCP is further improved by IIR filter in Generalized SCP (GSCP) proposed in [6, 9], which can achieve the best error resiliency among LP, SCP, and GSCP, due to the heavy filtering of IIR. In [7], GSCP is applied in H.264 scalable video coding (SVC).

Apparently the main target of RM is to improve the error resiliency; nevertheless there are still 2 additional challenges need consideration. 1) Coding efficiency overhead. Aiming for error resiliency, the reference modification is apparently an overhead in terms of compression efficiency, since the correlation between the reference and the coding block is reduced. Unfortunately, much of this kind of overhead was introduces in LP, SCP, and GSCP, although the error resiliency was improved. 2) Standard decoder compatibility. LP and GSCP change the standard decoding structure due to the reference synchronization of encoder and decoder, while SCP does not.

Aiming at these 2 challenges, in this paper a 1) low bit rate overhead based and 2) standard decoding compatible based RM method is proposed, which achieves the best trade-off between the error resiliency and coding efficiency. In the proposal, the modified reference is formed by an expected decoder reconstruction, thus unnecessary to change the standard decoding process for reference synchronization of encoder and decoder, and can achieve better error resiliency. During the formulation of expected decoder reconstruction, the BMA based EC at the decoder side is employed, in which the modified reference has more correlation to original reference, thus can reduce the bit 
rate overhead. Overall, the proposal provides the best trade-off among the error resiliency, coding efficiency, and standard compatibility.

\section{Overview of BMA based EC}

The Error Concealment (EC) is to recover the lost MB by the video redundancy. Simply copy the previous collocated MB for a lost MB is a basic method of EC. An advanced solution is BMA (boundary match algorithm) [2]. For each lost MB, BMA is utilized to recover the lost $m v$ (motion vector) from candidate $m v \mathrm{~s}\left(m v^{c a n} \mathrm{~s}\right)$ by minimizing the side match distortion $\left(D_{s m}\right)$ between the IN-MB and OUT-MB, see Fig. 1 and Eq. (1). The IN-MB is pointed by $m v^{c a n}$, which is the candidate $m v$ belonging to one of four available neighbors (top, bottom, left, and right) in current frame. (In Fig. 1, only top and left mvs are shown as available neighbors)

$D_{s m}$ is determined by the sum of absolute differences between boundary pixel $Y\left(m v^{c a n}\right)_{m}^{I N}$ at the predicted $\mathrm{MB}$ and $Y_{m}^{O U T}$ of the neighboring MB, shown in Eq. (1) ${ }^{1}$.

$$
\begin{aligned}
& D_{s m}=\frac{1}{M} \sum_{m=0}^{M-1}\left|Y\left(m v^{c a n}\right)_{m}^{I N}-Y_{m}^{O U T}\right| \\
& \text { where, } m v^{\text {can }} \in\left\{m v^{\text {top }}, m v^{\text {bottom }}, m v^{\text {left }}, m v^{\text {right }}\right\}
\end{aligned}
$$

$M$ is the total number of the available boundary pixels. Taking Fig. 1 as an example, $M=32$, since only 2 neighbors are available $(16 * 2)$. The winning prediction $m v\left(m v^{\text {win }}\right)$ is the one that minimizes the side match distortion $D_{s m}$ :

$$
m v^{\text {win }}=\underset{m v^{\text {can }} \in\left\{m v^{\text {top }}, m v^{\text {bottom }}, m v^{l e f t}, m v^{\text {right }}\right\}}{\operatorname{Arg~Min}} D_{s m}
$$

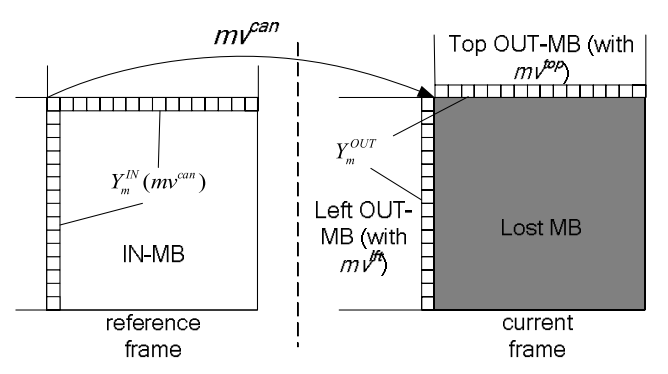

Fig. 1. BMA based EC

\section{Overview several methods for reference modification (RM)}

In conventional H.264 encoding system, the reference access is block by block whose size is $4 \times 4$ pixels. The raw block $B_{n}^{j}$ in frame $\mathrm{n}$ is encoded to a bit

\footnotetext{
${ }^{1}$ There is a notation rule needs explanation. In Fig. $1, Y_{m}^{I N}\left(m v^{c a n}\right)$ is the $m$-th pixel in the IN-MB, which is pointed by $m v^{c a n}$ in the reference frame. Such rule can be similarly applied in Eq. (8) in the rest parts.
} 
stream, and reconstructed to an encoder reconstruction $B_{r e c, n}^{j}$, which will be used as a reference block $B_{r e f, n}^{j}$ for the block $B_{n+1}^{i}$ encoding in the next frame $\mathrm{n}+1$, see Eq. (3). Here using different superscripts $i$ and $j$ means $B_{n+1}^{i}$ and its reference $B_{r e f, n}^{j}$ may not lay in the co-located position.

$$
B_{r e f, n}^{j}=B_{r e c, n}^{j}
$$

The reference used in RM approach is a modified version ${B^{\prime j}}_{r e f, n}$, which can be less vulnerable to channel errors. Several RM methods will be reviewed in this section.

\subsection{Leaky prediction (LP)}

In LP [4], the modified reference $B_{r e f, n}^{\prime j}$ can be formed by:

$$
B_{r e f, n}^{\prime j}=\alpha \cdot B_{r e c, n}^{j}+(1-\alpha) \cdot K
$$

where, $\alpha$ and $\mathrm{K}$ are the leaky factor and a constant respectively. $\alpha$ may take a value between 0 and 1 . It controls the trade off between coding efficiency and error resiliency. Typically, $\alpha$ locates in $(0.8,0.95)$, and can be optimized by analytical solutions [4]. $K$ is 128 , which usually takes on the mid range of pixel value [8].

Apparently the standard decoder needs consequent change to synchronize the reference, which is a drawback of LP. Another drawback is LP introduces a lot of bit rate overhead.

\subsection{Source channel prediction (SCP)}

In SCP [5], the expected decoder reconstruction $B_{d e c, n}^{j}$ is used for modified reference, rather than the encoder reconstruction $B_{r e c, n}^{j}$ in conventional encoder like Eq. (3).

Under the model of Bernoulli process, a weighted sum of reconstructed block $B_{r e c, n}^{j}$ and the recovered block $B_{r c v, n}^{j}$ is used as the formulation of the expected decoder reconstruction. Therefore, the modified reference can be formed by a FIR filter, in Eq. (5), where $p$ is packet loss rate. The recovered block $B_{r c v, n}^{j}$ is obtained by a recovery of a lost $B_{r e c, n}^{j}$. The recovery approach is simply copy the previous collocated block $B_{r e c, n-1}^{j}$, seen in Eq. (5).

$$
\begin{aligned}
& B_{r e f, n}^{\prime j}=\alpha \cdot B_{r e c, n}^{j}+(1-\alpha) \cdot B_{r c v, n}^{j}, \text { where } \alpha=1-p \\
& B_{r c v, n}^{j}=B_{r e c, n-1}^{j}
\end{aligned}
$$

Note that under the principle of using expected decoder reconstruction for reference (with the Bernoulli process model), the conventional decoder structure keeps no change.

In addition, compared to LP, SCP reduces the bit rate overhead, since the modified reference has more correlation to current encoding frame. 


\subsection{Generalized SCP (GSCP)}

In GSCP [6, 9], the modified reference can be formed by an IIR filter:

$$
B_{r e f, n}^{\prime j}=\alpha \cdot B_{r e c, n}^{j}+(1-\alpha) \cdot B_{r e f, n-1}^{\prime k}, \text { where } \alpha=1-p-H
$$

$H$ is a constant between 0.1 to 0.2 , and can be obtained by trial and error [6]. $B_{r e f, n-1}^{\prime k}$ is the modified reference block in frame $\mathrm{n}-1$ for encoding block $B_{n}^{j}$, whose reconstructed block is $B_{r e c, n}^{j}$. Here using different superscripts $j$ and $k$ means $B_{n}^{j}$ and its reference $B_{r e f, n-1}^{\prime k}$ may not lie in the co-located position.

GSCP is a generalized SCP, with flexible weighting of sum and modifies both encoder and decoder. GSCP doesn't perform under the principle of expected decoder reconstruction; therefore the decoding process is changed for synchronization of encoder and decoder.

As for error resiliency, since IIR filter in (6) generated heavier filtering than FIR in (5), GSCP may be more robust to errors. On the other hand, since less correlation with original frame is introduced, the coding efficiency is decreased.

\section{Proposed low bit rate overhead based RM approach}

The proposal is addressing the low bit rate overhead based RM, in which the reference is highly correlated to original reference. On the other hand, proposed RM still keeps the error control ability.

The proposal follows the principle of the expected decoder reconstruction $B_{d e c, n}^{j}$ of SCP. However, for the recovered block $B_{r c v, n}^{j}$, rather than simply copy the previous collocated block in SCP, we use BMA based EC to calculate it.

Using $B_{d e c, n}^{j}$ as the reference can achieve the minimal end to end distortion, ie., distortion of encoder input raw frame and decoder output reconstructed frame, see Fig. 2 (a). This was proved in mathematical way by [5] Here we want to explain it in intuitive way. It is well known that the quantization loss ( $\mathrm{Q}$ loss) is involved in reconstructed block $B_{r e c, n}^{j}$. On the other hand, besides Q loss, the transmission loss is also involved in decoded block $B_{d e c, n}^{j}$. Therefore, in error prone case, using expected decoder reconstruction can synchronize the reference at both encoder and decoder, thus results in better error resilience.

The expected decoder reconstruction is formulated by a weighted sum of the reconstructed block $B_{r e c, n}^{j}$ and the recovered block $B_{r c v, n}^{j}$, under the model of Bernoulli process. In Fig. 2(b), in order to encode a raw block

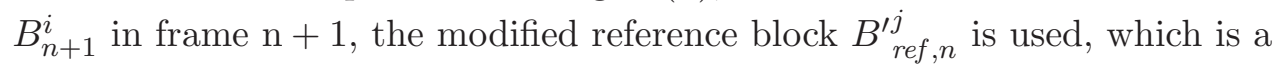
weighted sum of original reconstructed block $B_{r e c, n}^{j}$ and the recovered block $B_{r c v, n}^{j}$ in frame n, see Fig. 2 (b) and Eq. (7).

$$
\begin{aligned}
& B_{r e f, n}^{\prime j}=\alpha \cdot B_{r e c, n}^{j}+(1-\alpha) \cdot B_{r c v, n}^{j}, \text { where } \alpha=1-p \\
& B_{r c v, n}^{j}=\sum_{l=0}^{3} P_{r c v, n}^{j, l}, \text { where } P_{r c v, n}^{j, l} \in M B_{r c v, n}^{j, l}
\end{aligned}
$$




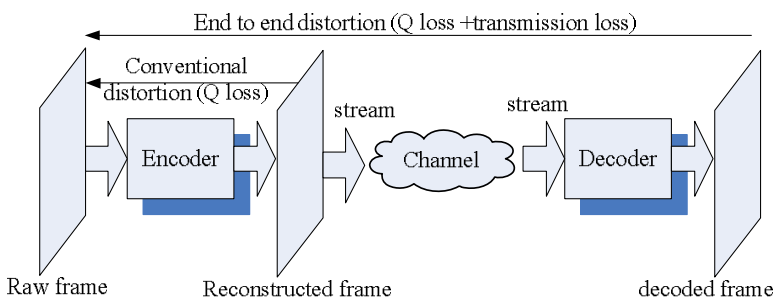

(a) End to end distortion

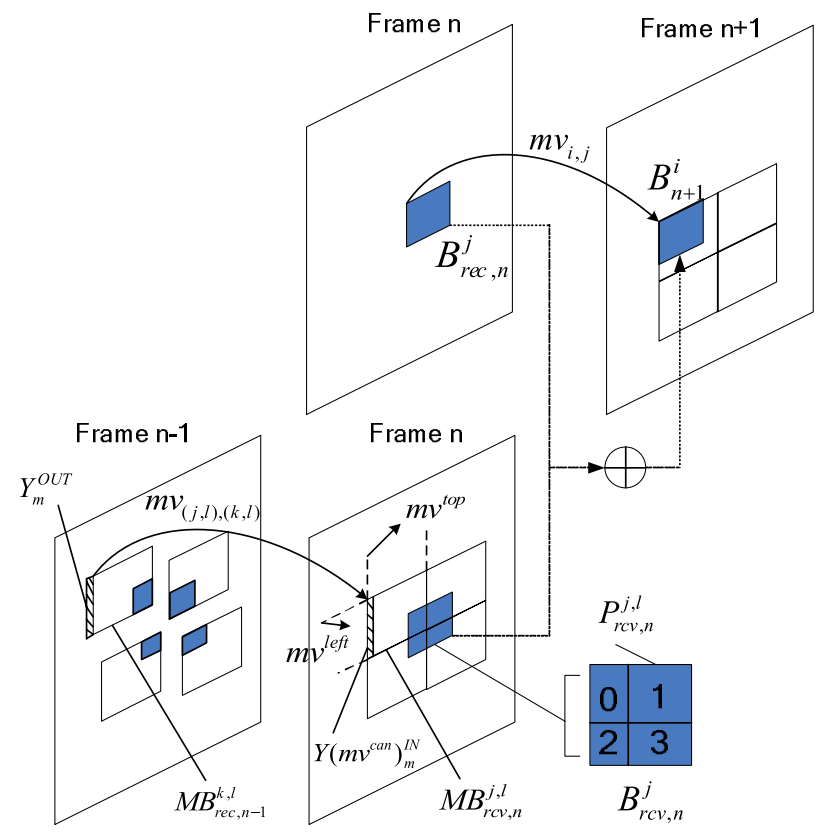

(b)Formulation of $B_{n+1}^{i}$ 's modified reference block $B_{r e f, n}^{{ }^{j}}$

Fig. 2. Illustration of proposal

In Eq. (7), $B_{r c v, n}^{j}$ is a recovery block for $B_{r e c, n}^{j}$, under the hypothesis that $B_{r e c, n}^{j}$ is lost during the transmission, and can be found by BMA based EC. $B_{r c v, n}^{j}$ may be divided up to 4 partitions $\left(P_{r c v, n}^{j, l},(l=0,1,2,3)\right)$ by the $16 \times 16 \mathrm{MB}$ boundaries. Each partition $P_{r c v, n}^{j, l}$ belongs to a MB $M B_{r c v, n}^{j, l}$ in frame n. (This can be seen in Fig. 2(b), and note that the size of $4 \times 4$ block $B_{r c v, n}^{j}$ is enlarged to make it more visible under the $16 \times 16 \mathrm{MB}$ size.) Therefore obtain $M B_{r c v, n}^{j, l}$ can consequently obtain $P_{r c v, n}^{j, l}$.

In order to get $M B_{r c v, n}^{j, l}$, BMA like way is used as follows. Suppose $M B_{r c v, n}^{j, l}$ is lost, it can be recovered by copy an appropriate MB $M B_{r e c, n-1}^{k, l}$ in frame $\mathrm{n}-1$ with minimal side match distortion $d_{s m}$. The appropriate $M B_{r e c, n-1}^{k, l}$ is pointed by a motion vector $m v_{(j, l),(k, l)}$, which can be formulated by BMA as Eq. (8). $Y\left(m v^{c a n}\right)_{m}^{I N}$ and $Y_{m}^{O U T}$ are the boundary pixels shown in Fig. 2 (b). Note that all 4 neighbors (top, bottom, left, and right) of $M B_{r c v, n}^{j, l}$ are available at the encoder side, therefore, $M=64(16 * 4)$.

$$
\begin{aligned}
& m v_{(j, l),(k, l)} \\
& =\underset{\text { can } \in\{\text { top }, \text { bot }, \text { left, right }\}}{\arg \operatorname{Min}}\left\langle D_{s m}=\frac{1}{64} \sum_{m=0}^{63}\left|Y\left(m v^{\text {can }}\right)_{m}^{I N}-Y_{m}^{\text {OUT }}\right|\right\rangle
\end{aligned}
$$

Compared with SCP, the proposal still uses expected decoder reconstruc- 
tion principle. However, rather than the collocated MB copy, the BMA makes the proposal achieve a more accurate expectation of decoder reconstruction. As a result, the proposal can achieve a better performance of error control. When compared with GSCP, which has the best error control performance in section 3, thanks to advanced BMA based EC, the proposal even achieves better performance than GSCP according to the simulation results in section 5 .

As for coding efficiency, since BMA based $B_{r c v, n}^{j}$ in proposal has more correlation to original reference block than co-located copy based $B_{r c v, n}^{j}$ in SCP, which has the best coding efficiency in section 3, the proposal can still achieve lower bit rate overhead than SCP.

For decoding compatibility, following the principle of the expected decoder reconstruction, the proposal makes no change at the decoder side, thus compatible to standard decoding.

\section{Experiments}

The proposed algorithm is evaluated by the H.264, with its reference software JM [4]. Our proposal is implemented at encoder. 30 frames for stefan, foreman, and football sequences in CIF format are encoded with baseline profile. I frame is inserted every $10 \mathrm{P}$ frames. Number of reference frames is set to 1. FMO with dispersed pattern is used. There is no change at the decoder. The packet is randomly lost. Packet loss rate (PLR) with 0.1, 0.2, and 0.3 are simulated.

We compared the performance of the proposal with other existing methods, ie., the original CODE with no reference modification ("H.264"), SCP, and GSCP. In GSCP, $\mathrm{H}=0.13$. For the decoding process, there is no modification for H.264, SCP, and the proposal, while there is an associate modification for GSCP to synchronize the reference with decoder. Note that here intra MB refreshment [6] is not combined together in GSCP, while in the experiment of $[6]$ it is combined.

Fig. 3 (a) shows the coding efficiency comparison when PLR $=30 \%$ for foreman and stefan. The bit rate is used to evaluate the coding efficiency. It can be seen that: 1) GSCP produces the highest bit rate, since the heaviest filter was used for reference modification. 2) H.264 has the lowest bit rate production, since there is no reference modification. 3) SCP produces the middle level between H.264 and GSCP. 4) Last but not least, proposal achieves almost the same bit rate with H.264, saves up to $200 \mathrm{Kbps}$ over GSCP, which means very low bit rate overhead was introduced compared to H.264. This confirms that the proposal is a low bit rate overhead approach. Note that the scale range of vertical axis is different for foreman and stefan in Fig. 3 (a).

Fig. 3 (b) shows the error resiliency comparison for different packet loss rates. It can be seen that, 1) each of SCP, GSCP, and proposal has a little higher PSNR than H.264, meaning better error control because of reference modification; 2) GSCP achieves a little higher PSNR than SCP since heavier 

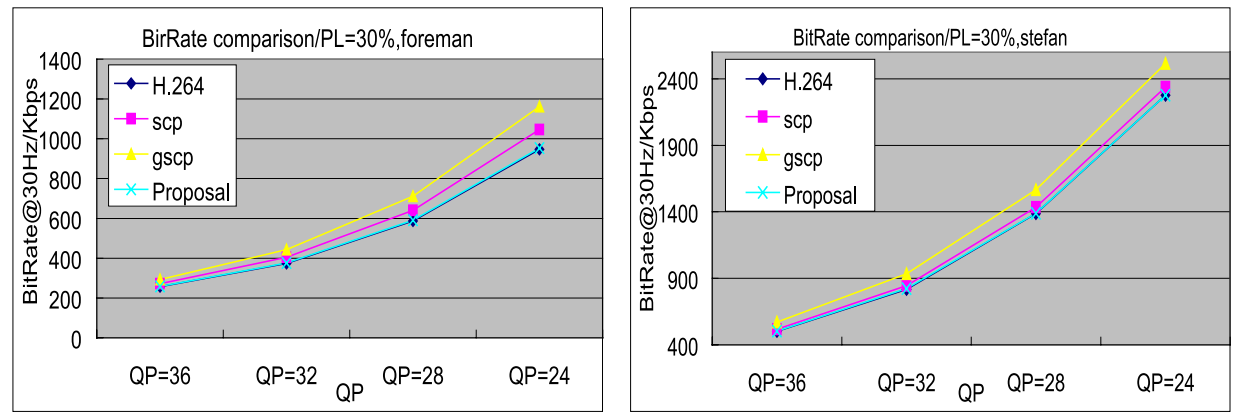

(a) Coding efficiency comparison
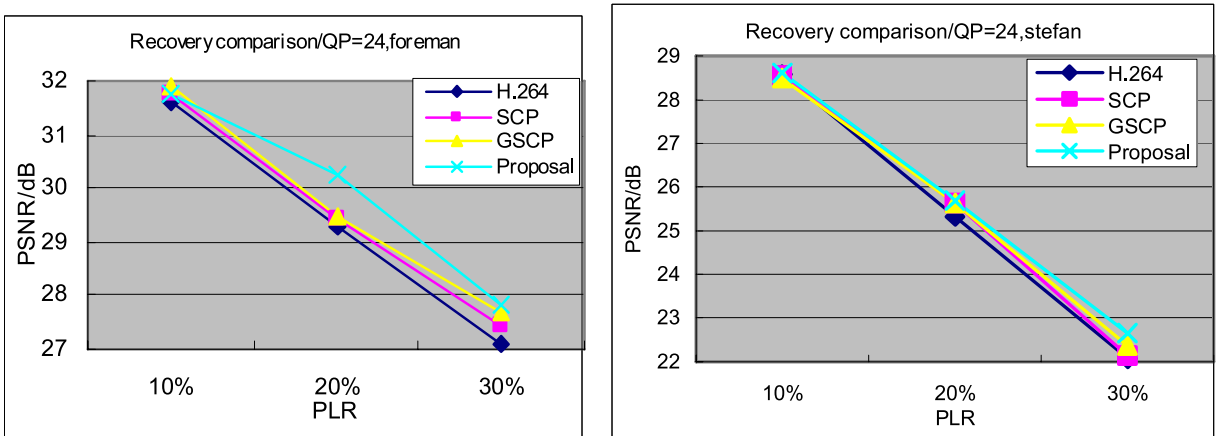

(b) Error resiliency comparison
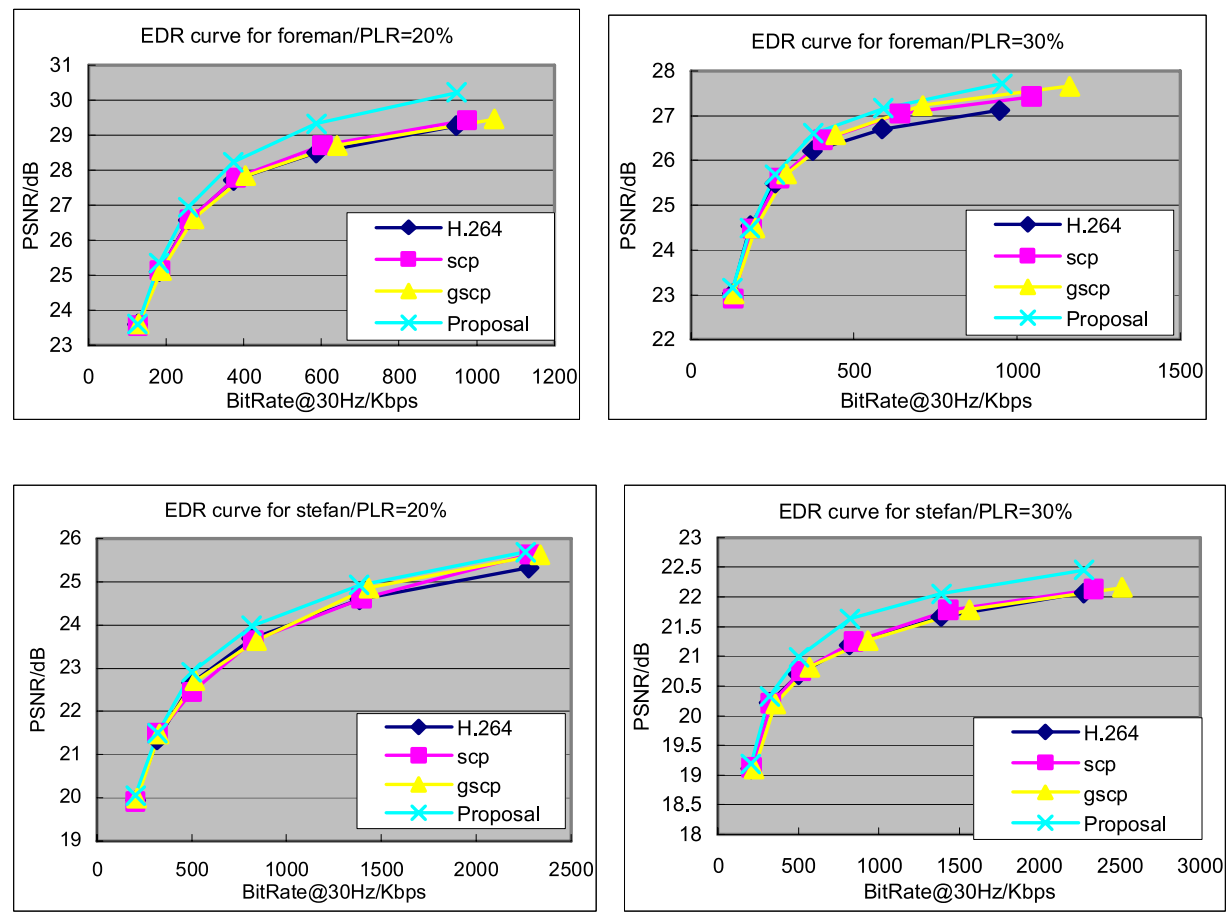

(c) Over all performance

Fig. 3. Experiments results

filtering was introduced; 3) Last but not least, proposal achieves the highest PSNR among them (up to $1 \mathrm{db}$ gain over H.264), which means the best error control performance was achieved. Note that here the PSNR is the end to end distortion.

As for overall comparison, ie., the trade off between the coding efficiency 
and error resiliency, the End to end rate Distortion to bit Rate (EDR) curve is used for evaluation, instead of conventional reconstruction distortion to bit rate curve. This is because the transmission loss is involved in end to end distortion. A curve lies on the upper position means better overall performance is achieved. Fig. 3 (c) shows the overall performance comparison results. It can be seen that the proposal achieves the best overall performance.

\section{Conclusion}

A low bit rate overhead based RM for H.264 is proposed in this paper, which still keeps the error resiliency. In the proposal, the modified reference is formed by an expected decoder reconstruction, thus unnecessary to change the standard decoding process, and can achieve better error resiliency. During the formulation of expected decoder reconstruction, the BMA based EC at the decoder side is employed, where the modified reference has more correlation to original reference, thus can reduce the bit rate overhead. Overall, the proposal provides the best trade off among the error resiliency, coding efficiency, and standard compatibility.

\section{Acknowledgments}

Authors would like to thank the discussion with Associate Professor Fabrice Labeau of McGill University Canada. We would also thank Global COE and CREST by JST Japan for funding this research. 